\title{
Simulations of Binary Particles Distributions in a Separated- Gasification Chemical Looping Combustion System
}

\author{
Xudong Wang, Yali Shao, Baosheng Jin \\ Key Laboratory of Energy Thermal Conversion and Control of Ministry of Education, School of Energy and Environment, \\ Southeast University \\ 2\# Sipailou, Nanjing, China \\ xdwang_seu@seu.edu.cn
}

\section{Extended Abstract}

Chemical looping combustion is a promising non-flame combustion technology which can separate $\mathrm{CO}_{2}$ during combustion process without extra energy penalty [1]. Previous, a separated-gasification chemical looping combustion system was designed and constructed, which consisted of a gasifier (GR), a reduction reactor (RR) and an air reactor (AR) [2]. The results of hot operation showed satisfying $\mathrm{CO}_{2}$ yield. In order to know the detailed gas-solid flow characteristics, a threedimensional computational fluid dynamics (CFD) model was adopted to predict the multiphase hydrodynamics. In the cold operation of this system, there are three phases, gas, coarse sand particle and oxygen carrier. The sand particle bubbles in GR while the oxygen carrier circulated between RR and AR. The detailed operation mechanism of this system can be found in our previous work [2]. This work mainly focused on the distributions of binary particles in the system under variable conditions.

First, the particle phases were regarded as fluids and the Eulerian-Eulerian model was developed coupled with kinetic theory of granular flow, which contained three phases, namely gas, sand and oxygen carrier. The parameters of sand and the OC particles are chosen as same as ref. [2]. The gas phase was employed as air. The geometry parameters were employed same as the experimental setup, where the diameters of GR, RR and AR were $50 \mathrm{~mm}, 34 \mathrm{~mm}$ and $530 \mathrm{~mm}$ while the heights were $500 \mathrm{~mm}, 6500 \mathrm{~mm}$ and $600 \mathrm{~mm}$. Considering the computation complexity and accuracy, a medium grid was chosen for the following simulation works.

Then, the simulations were conducted under variable conditions. The gas amount in RR was changed, which were 20.38, $22.32,24.26,26.20$ and $28.14 \mathrm{~m}^{3} / \mathrm{h}$. Under these conditions, the flow behaviours of gas and sand particle in GR and the flow mechanisms of gas and oxygen carrier were investigated.

Based on the simulation results, the distributions of the sand in GR and oxygen carrier in RR were obtained. The nonuniformity of particle was described using the standard deviation of solid fraction, $\sigma_{p}$. The variation of $\sigma_{p}$ was further fitted as the function of $N r$ and axial position. Results showed that the $\sigma_{p}$ decreased with the height of the RR. The $\sigma_{p}$ near OC return spot was largest in each test. The increase of the gas amount would cause the decrease of the $\sigma_{p}$ due to its sufficient fluidizing capability. In the well-developed section of RR, the $\sigma_{p}$ kept near constant.

\section{References}

[1] X. Wang, X. Wang, S. Zhang, Z. Kong, Z. Jin, Y. Shao, B. Jin, "Test Operation of a Separated-Gasification Chemical Looping Combustion System for Coal," Energ. Fuel., vol. 32, no. 11, pp. 11411-11420, 2018.

[2] J. Dong and D. Gidaspow, "A bubbling fluidization model using kinetic theory of granular flow," AICHE. J., vol. 36, no. 4, pp. 523-538, 1990. 